\title{
On the light quark mass effects in Higgs boson production in gluon fusion
}

\author{
Kirill Melnikov ${ }^{a}$ and Alexander Penin ${ }^{b, a}$ \\ ${ }^{a}$ Institut für Theoretische Teilchenphysik, \\ Karlsruhe Institute of Technology, 76128 Karlsruhe, Germany \\ ${ }^{b}$ Department of Physics, University of Alberta, \\ Edmonton, Alberta T6G 2J1, Canada \\ E-mail: kirill.melnikov@kit.edu, penin@ualberta.ca
}

\begin{abstract}
Production of Higgs bosons at the LHC is affected by the contribution of light quarks, that mediate the $g g \rightarrow H g$ transition. Although their impact is suppressed by small Yukawa couplings, it is enhanced by large logarithms of the ratio of the Higgs boson mass or its transverse momentum to light quark masses. We study the origin of this enhancement, focusing on the abelian corrections to $g g \rightarrow H g$ amplitudes of the form $\left(C_{F} \alpha_{s} \mathcal{L}^{2}\right)^{n}$, where $\mathcal{L} \in\left\{\ln \left(s / m_{b}^{2}\right), \ln \left(p_{\perp}^{2} / m_{b}^{2}\right)\right\}$. We show how these non-Sudakov double logarithmic terms can be resummed to all orders in the strong coupling constant. Interestingly, we find that the transverse momentum dependence of these corrections is very weak due to a peculiar cancellation between different logarithmic terms. Although the abelian part of QCD corrections is not expected to be dominant, it can be used to estimate missing higher-order corrections to light quark contributions to Higgs boson production at the LHC.
\end{abstract}

Keywords: Higgs Physics, Perturbative QCD, Resummation

ARXIV EPRINT: 1602.09020 


\section{Contents}

1 Introduction 1

2 Setup and notations 3

3 One-loop helicity amplitudes in the double logarithmic approximation 5

4 Two-loop helicity amplitudes in the double logarithmic approximation 9

5 Resummation of the abelian double logarithmic contributions 12

6 Double logarithmic corrections to the differential cross section 13

7 Conclusion $\quad 15$

$\begin{array}{ll}\text { A Polarization vectors } & 17\end{array}$

\section{Introduction}

The Higgs boson discovered at the LHC by ATLAS and CMS collaborations almost four years ago $[1,2]$ is a mysterious particle. Indeed, it seems to fit perfectly into the Standard Model (SM) of particle physics and its mass is numerically close to the weak scale $v$. However, the mechanism that would tie these two quantities together in a more general theory requires presence of other, relatively light, particles that couple to the Higgs boson. Such particles have not been observed so far and limits on their masses gradually become so tight that the "natural" relation $m_{H} \sim v$ is endangered. Further exploration of Higgs boson properties, including its couplings and quantum numbers, will be essential for understanding to what extent the observed particle is indeed described by the Standard Model and, hopefully, for discovering clues as to what the mass scale of physics beyond the Standard Model can actually be.

An important observable in Higgs physics is the Higgs boson transverse momentum distribution. There are several reasons for that. On one hand, precise knowledge of the Higgs boson $p_{\perp}$-distribution is important for understanding jet-vetoed cross sections and, more generally, observables subject to experimental constraints. The uncertainties in modeling the $p_{\perp}$-distribution affect values of the Higgs coupling constants extracted from such fiducial quantities. Since the total inclusive Higgs boson production cross section is currently known through next-to-next-to-next-to-leading order in perturbative QCD [3], the uncertainty in the Higgs $p_{\perp}$-distribution may become the dominant one when future experimental data is confronted with theoretical predictions for the Higgs boson production. 
Further motivation for the precise description of the Higgs boson $p_{\perp}$-spectrum comes from the observation that the $p_{\perp}$-distribution is, potentially, a good observable for detecting relatively light $\left(m \sim m_{H}\right)$ colored particles that couple to the Higgs boson [4]. Indeed the contribution of a particle with the mass $m \sim m_{H}$ to the Higgs boson production in gluon fusion is almost independent of $p_{\perp}$ for $p_{\perp}<m$ while for $p_{\perp}>m$ it rapidly decreases. Thus the $p_{\perp}$-distribution of Higgs bosons or jets recoiling against it, may serve as a sensitive probe of this type of physics beyond the Standard Model.

High-precision theoretical predictions for Higgs boson $p_{\perp}$-distribution within the Standard Model are necesary to pursue this program [5-8]. Unfortunately, despite significant progress in understanding the Higgs $p_{\perp}$-spectrum in recent years, the overall situation is unsatisfactory. The main challenge is to describe the bottom quark contribution to the Higgs boson production in gluon fusion at moderate values of the transverse momentum. Indeed, the $g g \rightarrow H$ transition in the Standard Model is dominated by the top-quark loop, thanks to the large Higgs-top Yukawa coupling. Since the top quark mass is large compared to the Higgs mass, it is possible to integrate out the top quark and describe the Higgs production at sufficiently low transverse momentum in the effective field theory with a local $g g H$ interaction. This reduces the number of loops in perturbative computations by one and allows us to push them to very high orders in QCD perturbation theory. Within this approximation, the Higgs $p_{\perp}$-distribution has been evaluated through next-to-next-toleading order at high $p_{\perp}<m_{t}[9,10]$ and to next-to-next-to-leading logarithmic accuracy at low $p_{\perp}<m_{H}[11,12]{ }^{1}$

At the same time understanding the bottom-quark contribution to $g g \rightarrow H g$ turned out to be more involved. ${ }^{2}$ Indeed, since $m_{b} \sim 4.2 \mathrm{GeV}$, the point-like approximation for the bottom quark contribution to $g g H$ vertex is only valid for tiny transverse momenta $p_{\perp}<m_{b}$. In a broader and more interesting momentum region $p_{\perp}>m_{b}$, the local vertex approximation for the bottom quark-mediated $g g H$ interaction is invalid and we must deal with the computation of complicated box diagrams with internal masses. Calculation of such diagrams at two and more loops is beyond the reach of existing computational techniques. ${ }^{3}$ As the result, the $g g \rightarrow H g$ amplitudes for $p_{\perp}>m_{b}$ are only known in the leading (one-loop) approximation.

The bottom quark contribution to Higgs boson production is small, compared to the contribution of the top quark. However, it is still relevant phenomenologically because of the high precision of forthcoming experimental measurements of the Higgs-gluon coupling and because the bottom quark contribution is dynamically enhanced. Indeed, although the coupling of the bottom quark to the Higgs boson is small compared to the Higgs-top coupling, the $n$-loop bottom quark contribution to $g g \rightarrow H g$ is enhanced by two powers of large logarithms per one power of $\alpha_{s}$, i.e. $\mathcal{O}\left(\alpha_{s}^{n} \mathcal{L}^{2 n}\right)$, where $\mathcal{L} \in\left\{\ln \left(m_{H}^{2} / m_{b}^{2}\right), \ln \left(p_{\perp}^{2} / m_{b}^{2}\right)\right\}$. For relevant values of the transverse momentum $p_{\perp} \sim 30 \mathrm{GeV}$ and the Higgs boson mass $m_{H}=125 \mathrm{GeV}$, these logarithms can be numerically quite large $\mathcal{L}^{2} \sim 20-50$. In fact, the

\footnotetext{
${ }^{1}$ For a recent discussion and further references see ref. [13].

${ }^{2}$ Contributions of even lighter quarks are negligible.

${ }^{3}$ For a recent progress in the next-to-next-to-leading order analysis of the bottom quark mass effects in $g g \rightarrow H$ production see ref. [14].
} 
magnitude of the double logarithmic corrections suggests that the all-order resummation may be necessary.

The origin of these logarithmically enhanced terms is currently not well understood. Although their double logarithmic nature suggests a mechanism similar to the Sudakov enhancement [15], as we explain below the mass suppression of the amplitude $\mathcal{M}_{g g \rightarrow H g} \sim m_{b}^{2}$ makes such an interpretation problematic. Contribution of bottom quarks to the Higgs boson production in gluon fusion was discussed in refs. [16-18] in the context of $p_{\perp^{-}}$ resummation. There it was pointed out that the standard technology of $p_{\perp}$-resummation only applies for $p_{\perp}<m_{b}$, while for larger values of $p_{\perp}$ it is incomplete. The authors of refs. [16-18] then used differences between various resummation prescriptions to estimate the uncertainty in the Higgs $p_{\perp}$-distribution, caused by unknown higher-order QCD corrections to the bottom quark contribution.

The goal of this paper is to make a step towards a better understanding of the origin of double logarithmic corrections to the Higgs boson production, their computation in the two-loop approximation and to their resummation. Since these tasks are very challenging, we restrict our analysis to abelian QCD corrections, i.e. corrections associated with the abelian color factor $C_{F}^{n}$ in the $n$-th order of QCD perturbation theory. Note that the abelian radiative corrections are generated by the coupling of virtual gluons to massive offshell quarks. As a consequence, these corrections are infra-red finite on their own, so that physical results can be obtained without the need to consider processes with additional soft and collinear radiation.

The paper is organized as follows. In the next section we introduce our notation. In section 3 we describe evaluation of one-loop double logarithmic corrections to the bottom quark contribution to $g g \rightarrow H g$ helicity amplitudes. In section 4 we extend this analysis to two loops. In section 5 we show how these logarithmic corrections can be resummed to all orders in the strong coupling constant. Numerical estimates of the corrections are given in section 6 . We conclude in section 7 .

\section{Setup and notations}

We consider the Higgs boson production in the process $g g \rightarrow H g$ mediated by the bottom quark loop. The Higgs boson has a non-vanishing transverse momentum. The particle momenta are assigned in the following way

$$
g\left(p_{1}\right)+g\left(p_{2}\right) \rightarrow g\left(p_{3}\right)+H\left(p_{H}\right) .
$$

Our goal is to find the double logarithmic contributions to helicity amplitudes in a kinematic situation where the energy of the final state gluon $E_{3}$ is much smaller than the energies of the colliding gluons $E_{1,2}$ and the Higgs boson mass. At the same time, we consider $E_{3}$ to be much larger than the mass of the quark that mediates the $g g \rightarrow H$ transition. When written in terms of kinematic invariants, these conditions become

$$
m_{b}^{2} \ll t, u \ll s, m_{H}^{2},
$$


where $s=\left(p_{1}+p_{2}\right)^{2}, t=\left(p_{1}-p_{3}\right)^{2}, u=\left(p_{2}-p_{3}\right)^{2}$. Eq. (2.2) in particular implies that $m_{b}^{2} \ll p_{\perp}^{2} \ll t, u$, where $p_{\perp}^{2}=t u / s$ is the square of the transverse momentum of the Higgs boson or the gluon in the final state.

To illustrate this kinematic situation further, consider the production of a Higgs boson through a bottom quark loop accompanied by an emission of a soft gluon. We take $m_{b}=$ $4.2 \mathrm{GeV}, \sqrt{s} \approx m_{H}, p_{\perp} \approx 20 \mathrm{GeV}$ and assume central production (small rapidity), so that $E_{3} \approx p_{\perp}$. Numerically we find

$$
\frac{m_{b}}{E_{3}} \sim \lambda, \quad \frac{E_{3}}{E_{1}} \sim \lambda,
$$

with $\lambda \sim 0.25$. We consider $\lambda$ to be a small parameter and adopt the scaling rules eq. (2.3). In the limit $\lambda \rightarrow 0$ the $g g \rightarrow H g$ amplitude develops the $1 / \lambda$ singularity, characteristic to the soft gluon emissions; this allows us to write the perturbative series for the amplitude in the following way

$$
\mathcal{M}_{g g \rightarrow H g}=\frac{g_{s}}{\lambda} \sum_{n=1}^{\infty} C_{n} \alpha_{s}^{n} \ln ^{2 n}(\lambda)+\ldots .
$$

In eq. (2.4), we neglected all terms that are less singular than $\lambda^{-1} \alpha_{s}^{n} \ln ^{2 n} \lambda$ in the $\lambda \rightarrow 0$ limit. We are interested in the abelian part of the coefficients $C_{n}$, which determine the double logarithmic approximation for the amplitude. The leading-order coefficient $C_{1}$ is well-known and can be extracted from the one-loop result for the $g g \rightarrow H g$ amplitude [19]. In what follows, we explain how to obtain this coefficient without following the standard route of a one-loop computation. We then compute the two-loop coefficient $C_{2}$ and generalize the result to arbitrary $n$.

We begin by fixing the notation for helicity amplitudes. There are eight helicity amplitudes that are needed to describe $g_{1} g_{2} \rightarrow H g_{3}$ process. However, when the gluon $g_{3}$ is soft, the Higgs boson is effectively produced in the collision of two energetic gluons $g_{1}$ and $g_{2}$. This can only happen when helicities of these gluons are equal. The constraint $\lambda_{1}=\lambda_{2}$ leaves us with four helicity amplitudes which are pair-wise related by the parity conjugation. We take $M_{+++}$and $M_{++-}$as the two independent amplitudes that we need to compute.

It is convenient to write the amplitudes in such a way that their spin-helicity structure in the soft limit is factored out, and the remaining part only depends on the Mandelstam invariants of the process

$$
\begin{aligned}
& M_{+++}^{\text {soft }}=-g_{s} \sqrt{2} f^{a_{1} a_{2} a_{3}} \frac{g_{s}^{2} g_{y} m_{b}}{16 \pi^{2}} \frac{\langle 12\rangle^{2}}{[12]\langle 23\rangle\langle 13\rangle} A_{+++}\left(t, u, m_{H}^{2}, m_{b}^{2}\right), \\
& M_{++-}^{\text {soft }}=-g_{s} \sqrt{2} f^{a_{1} a_{2} a_{3}} \frac{g_{s}^{2} g_{Y} m_{b}}{16 \pi^{2}} \frac{\langle 12\rangle}{[23][13]} A_{++-}\left(t, u, m_{H}^{2}, m_{b}^{2}\right) .
\end{aligned}
$$

Two helicity-dependent form factors $A_{++ \pm}$are given by the series in the strong coupling constant

$$
A_{++ \pm}=A_{++ \pm}^{(0)}+\left(\frac{\alpha_{s}}{2 \pi}\right)\left(C_{F} A_{++ \pm}^{(1 A)}+C_{A} A_{++ \pm}^{(1 N A)}\right)+\mathcal{O}\left(\alpha_{s}^{2}\right),
$$

where the abelian and non-abelian parts are separated. Our goal is to compute abelian contributions at two loops and beyond. 


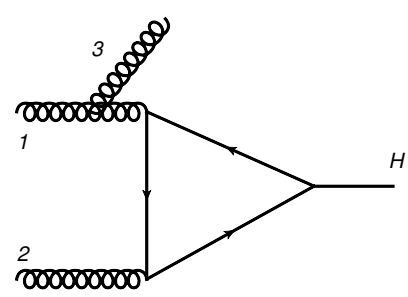

a)

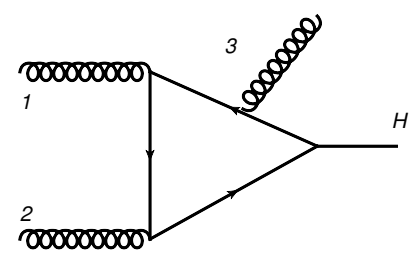

b)

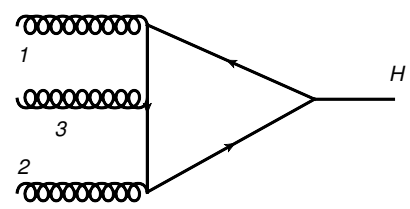

c)

Figure 1. One-loop diagrams representing the leading order bottom quark contribution to $g g \rightarrow H g$ process. Symmetric diagrams corresponding to the opposite direction of the quark flow and to the soft emissions off the opposite gluon/quark line are not shown.

\section{One-loop helicity amplitudes in the double logarithmic approximation}

In this section, we will study the double logarithmic contributions to the one-loop $g g \rightarrow H g$ amplitude mediated by a quark of mass $m$. A well-known example of a double logarithmic enhancement is provided by the Sudakov logarithms [15]. However, the situation with $g g \rightarrow H g$ is different. Indeed, as we will show, in contrast to the Sudakov logarithms [15] associated with the radiation of soft virtual gauge bosons by highly energetic on-shell charged particles, the double logarithmic enhancement of the $g g \rightarrow H g$ amplitude is caused by a soft quark exchange. Such non-Sudakov double logarithms are typical for amplitudes that are mass-suppressed at high energy [20-22]. Since physics of these non-Sudakov double logarithmic corrections is not well-known, we begin by discussing the one-loop case in detail.

In total, there are ten one-loop Feynman diagrams contributing to the leading order $g g \rightarrow H g$ amplitude, figure 1. However, up to differences in color factors that ensure that the final result is proportional to structure constants $f^{a b c}$ of the gauge group $\mathrm{SU}(3)$, diagrams that differ only by the direction of the quark flow in the loop give identical contributions. The number of relevant diagrams can be further reduced by a judicious choice of gluon polarization vectors. Indeed, each polarization vector can be chosen to satisfy two transversality conditions. It is convenient to require

$$
\epsilon_{i} \cdot p_{i}=0, \quad i \in\{1,2,3\}, \quad \epsilon_{1} \cdot p_{2}=0, \quad \epsilon_{2} \cdot p_{1}=0, \quad \epsilon_{3} \cdot p_{2}=0
$$

Explicit expressions for polarization vectors satisfying eq. (3.1) in terms of spinor products are given in appendix. Emission of a soft gluon $g_{3}$ off the gluon or quark line carrying large momentum $p_{i}$ can be described by an effective vertex proportional to $p_{i} \cdot \epsilon_{3}$. Thus the condition $\epsilon_{3} \cdot p_{2}=0$ ensures that there are no soft gluon emissions off the gluon and quark lines carrying the external momentum $p_{2}$. As the result, only diagrams shown in figure 1 need to be considered.

To determine the double logarithmic asymptotic behavior of the amplitude we follow the original method of ref. [15]. We start by evaluating the diagram figure 1a together with the diagram with the opposite direction of the quark flow. By calculating the trace we find that the diagram is proportional to $m_{b}$. The mass suppression is caused by the fact that the Higgs-quark interaction flips quark helicity. Since strong interactions conserve helicity 
in the massless limit, the mass term provides the required second helicity flip in the quark loop. This helicity flip is caused by a mass term of the soft quark propagator

$$
\frac{\hat{l}+m_{b}}{l^{2}-m_{b}^{2}} \rightarrow \frac{m_{b}}{l^{2}-m_{b}^{2}}
$$

It follows from eq. (3.2) that once the mass term is selected, the soft quark propagator becomes a propagator of a scalar particle, which is sufficiently singular at small momenta to develop a double logarithmic contribution. We note that if the mass term is taken from the quark propagator that carries large momentum, the double logarithmic contribution does not develop because the soft quark propagator $1 / \hat{l}$ is insufficiently singular. By virtue of a similar argument, the soft loop momentum $l$ can often be neglected in the numerators of contributing diagrams, since we are only interested in the leading logarithmic enhancement. We note that the last feature is not generic (see e.g. the analysis of the diagram figure 1c below). With all these simplifications it is straightforward to derive contributions of the diagram figure 1a to the helicity-dependent form factors. They read

$$
A_{++ \pm}^{(0), 1 a}=-32 i \pi^{2} s C\left(s, t, m_{b}^{2}\right)
$$

where

$$
C\left(s, t, m_{b}^{2}\right)=\int \frac{\mathrm{d}^{4} l}{(2 \pi)^{4}} \frac{1}{\left(l^{2}-m_{b}^{2}\right)\left(\left(p_{1}-p_{3}-l\right)^{2}-m_{b}^{2}\right)\left(\left(p_{2}+l\right)^{2}-m_{b}^{2}\right)}
$$

is the three-point function with two of its legs off-shell. To compute $C\left(s, t, m_{b}^{2}\right)$ in the double logarithmic approximation, we follow ref. [15] and introduce the Sudakov parametrization of the virtual momentum $l=\alpha p_{1}+\beta p_{2}+l_{\perp}$. We integrate over the transverse momentum components $l_{\perp}$ by taking the residue of the soft quark propagator pole

$$
\frac{1}{l^{2}-m_{b}^{2}+i 0} \rightarrow-i \pi \delta\left(l^{2}-m_{b}^{2}\right)=-i \pi \delta\left(s \alpha \beta-l_{\perp}^{2}-m_{b}^{2}\right)
$$

This allows for a symmetric treatment of the soft and collinear parts of the double logarithmic contribution. The two remaining propagators in eq. (3.4) become

$$
\frac{1}{\left(p_{2}-l\right)^{2}-m_{b}^{2}} \rightarrow \frac{1}{s \alpha}, \quad \frac{1}{\left(p_{1}-p_{3}-l\right)^{2}-m_{b}^{2}} \rightarrow \frac{1}{t-\beta s}
$$

To obtain the double logarithmic contribution we require both $\alpha$ and $\beta$ integrations to be logarithmic. This requirement is automatically satisfied for the integration over $\alpha$. At the same time the integration over $\beta$ is logarithmic only for $\beta>|t| / s$. Hence, in the double logarithmic approximation eq. (3.4) reduces to

$$
C\left(s, t, m_{b}^{2}\right) \approx \frac{i}{16 \pi^{2} s} \int_{m_{b}^{2} / s}^{1} \frac{\mathrm{d} \alpha}{\alpha} \int_{|t| / s}^{1} \frac{\mathrm{d} \beta}{\beta} \theta\left(\alpha \beta-m_{b}^{2} / s\right),
$$

where the intervals $|t| / s<\beta<1$ and $m_{b}^{2} / s<\alpha<1$ are determined by the effective infrared and ultraviolet cutoffs of the logarithmic integral, and additional kinematic constraint $\alpha \beta>$ $m_{b}^{2} / s$ ensures that the pole of the soft quark propagator is in the integration domain. It is 
convenient to factor out the large logarithm $L=\ln \left(m_{b}^{2} / s\right) \approx \ln \left(m_{b}^{2} / m_{H}^{2}\right)$ and introduce the normalized variables $\eta=\ln \alpha / L$ and $\xi=\ln \beta / L$. By using eqs. (3.3), (3.4), (3.7) we find

$$
A_{++ \pm}^{(0), 1 a}= \pm 2 L^{2} \int_{0}^{1-\tau_{t}} \mathrm{~d} \eta \int_{0}^{1-\eta} \mathrm{d} \xi= \pm L^{2}\left(1-\tau_{t}^{2}\right)
$$

where $\tau_{t}=\ln \left(m_{b}^{2} /|t|\right) / L$.

Next, we consider the diagram figure $1 \mathrm{~b}$. To compute this diagram in the double logarithmic approximation, we again pick up a mass term from the soft quark in the $t$-channel and neglect the momenta $l$ and $p_{3}$ everywhere in the numerator. Then the contribution of the diagram figure $1 \mathrm{~b}$ reduces to

$$
A_{++ \pm}^{(0), 1 b}=-16 i \pi^{2} t s D\left(s, t, m_{H}^{2}, m_{b}^{2}\right)
$$

where $D\left(s, t, m_{H}^{2}, m_{b}^{2}\right)$ is the four-point integral

$$
D=\int \frac{\mathrm{d}^{4} l}{(2 \pi)^{4}} \frac{1}{\left.\left(l^{2}-m_{b}^{2}\right)\left(\left(p_{1}-l\right)^{2}-m_{b}^{2}\right)\left(p_{1}-p_{3}-l\right)^{2}-m_{b}^{2}\right)\left(\left(p_{2}+l\right)^{2}-m_{b}^{2}\right)} .
$$

We use the same Sudakov parametrization $l=\alpha p_{1}+\beta p_{2}+l_{\perp}$ as before. Upon inspecting the infrared structure of eq. (3.10) we find that the double logarithmic contribution can only be obtained when the propagator

$$
\frac{1}{\left(p_{1}-p_{3}-l\right)^{2}-m_{b}^{2}} \approx \frac{1}{t-\beta s}
$$

becomes independent of $\beta$. This leads to a constraint $\beta<|t| / s$. The logarithmic integration intervals become $m_{b}^{2} / s<\alpha<1, m_{b}^{2} / s<\beta<|t| / s$ and we obtain

$$
A_{++ \pm}^{(0), 1 b}= \pm L^{2} \int_{1-\tau_{t}}^{1} \mathrm{~d} \eta \int_{0}^{1-\eta} \mathrm{d} \xi= \pm L^{2} \frac{\tau_{t}^{2}}{2}
$$

We will now discuss the diagram shown in figure 1c where the gluon is emitted off the soft quark line. Similar to the previous case we deal here with the box diagram and need to "remove" one of its propagators to obtain the proper (logarithmic) scaling of the integrand. In fact, the underlying box diagram has two non-overlapping momenta regions that lead to a double logarithmic enhancement. These regions are characterized by the choice of the soft momentum in the diagram. Indeed, we can choose the soft momentum $l$ in such a way that the momentum of the emitted gluon $p_{3}$ flows through the lower (upper) half of the quark loop figure $1 \mathrm{c}$ in region I (II), respectively. Consider region I and choose the momentum decomposition $l=\alpha p_{1}+\beta p_{3}+l_{\perp}$. After omitting irrelevant terms, the quark propagators become

$$
\begin{aligned}
\frac{\hat{l}+m_{b}}{l^{2}-m_{b}^{2}} & \rightarrow-i \pi m_{b} \delta\left(|t| \alpha \beta-l_{\perp}^{2}-m_{b}^{2}\right), \\
\frac{\hat{p}_{1}-\hat{l}+m_{b}}{\left(p_{1}-l\right)^{2}-m_{b}^{2}} & \rightarrow \frac{\hat{p}_{1}}{t \beta},
\end{aligned}
$$




$$
\begin{aligned}
\frac{\hat{p}_{3}-\hat{l}+m_{b}}{\left(p_{3}-l\right)^{2}-m_{b}^{2}} & \rightarrow \frac{\hat{p}_{3}-\alpha \hat{p}_{1}}{t \alpha}, \\
\frac{\hat{p}_{2}+\hat{p}_{3}-\hat{l}+m_{b}}{\left(p_{2}-p_{3}+l\right)^{2}-m_{b}^{2}} & \rightarrow \frac{\hat{p}_{2}}{u+s \alpha} .
\end{aligned}
$$

It follows from eqs. (3.13), (3.14), (3.15), (3.16) that the double logarithmic contribution can be obtained in two different ways: (i) for $\alpha<|u| / s$ only the $p_{3}$ term in the numerator of eq. (3.15) should be kept (the "scalar" contribution) and (ii) for $\alpha>|u| / s$ the $\alpha p_{1}$ term should be taken from the numerator in eq. (3.15) to cancel an extra power of $\alpha$ that appears in the denominator of eq. (3.16) in this limit (the "vector" contribution). ${ }^{4}$ These two contributions are proportional to

$$
N_{\lambda_{1}, \lambda_{2}, \lambda_{3}}^{s}=\frac{\operatorname{Tr}\left[\hat{\epsilon}_{3} \hat{\epsilon}_{1} \hat{p}_{1} \hat{p}_{2} \hat{\epsilon}_{2} \hat{p}_{3}\right]}{2 t u}
$$

and

$$
N_{\lambda_{1}, \lambda_{2}, \lambda_{3}}^{v}=\frac{\operatorname{Tr}\left[\hat{\epsilon}_{3} \hat{\epsilon}_{1} \hat{p}_{1} \hat{p}_{2} \hat{\epsilon}_{2} \hat{p}_{1}\right]}{2 t s},
$$

respectively. By calculating traces and using explicit expressions for the polarization vectors given in appendix, we find the following results

$$
\begin{array}{ll}
N_{+,+,+}^{s}=0, & N_{++-}^{s}=\frac{\sqrt{2}\langle 12\rangle}{[13][23]}, \\
N_{+,+,+}^{v}=\frac{\sqrt{2}\langle 12\rangle^{2}}{[12]\langle 23\rangle\langle 13\rangle}, & N_{++-}^{v}=-\frac{\sqrt{2}\langle 12\rangle}{[13][23]},
\end{array}
$$

Note that the vector integral has the usual tensor structure of a color-dipole emission which gives $A_{+++}=-A_{++-}$, similar to all other diagrams. At the same time, the tensor structure of the scalar integral corresponds to the three-gluon configuration described by a local gauge invariant operator

$$
G_{\mu \nu}^{a} G_{\nu \lambda}^{b} G_{\lambda \mu}^{c} f^{a b c}
$$

which does not contribute to the all-plus helicity amplitude.

By crossing symmetry, the scalar contributions of the momentum regions I and II are equal. Therefore, the total scalar contribution of the diagram figure $1 \mathrm{c}$ can be written in terms of the double logarithmic integral over the interval $m_{b}^{2} /|t|<\alpha<|u| / s, m_{b}^{2} /|t|<\beta<$ 1 that originates from region I. This gives

$$
A_{++-}^{(0), 1 c, s}=-2 L^{2} \int_{1-\tau_{u}}^{\tau_{t}} \mathrm{~d} \eta \int_{0}^{\tau_{t}-\eta} \mathrm{d} \xi=-L^{2}\left(1-\tau_{t}-\tau_{u}\right)^{2}
$$

whereas $A_{+++}^{(0), 1 c, s}=0$. At the same time, the vector contribution of region II vanishes due to our choice of the polarization vector for the gluon $g_{3}, p_{2} \cdot \epsilon_{3}=0$. As the result, the total

\footnotetext{
${ }^{4}$ We refer to this contribution as "vector" because it originates from a term in the numerator of the diagram figure 1c which is linear in the soft loop momentum $l$.
} 


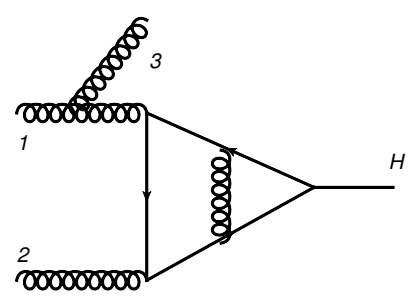

a)

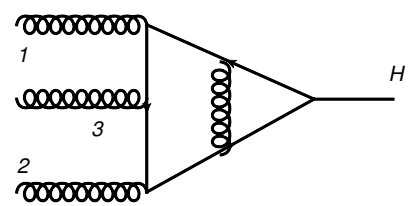

d)

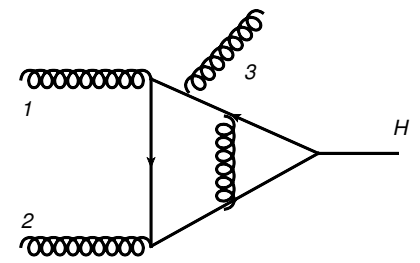

b)

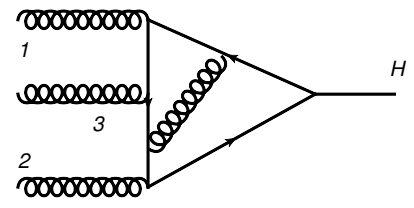

e)

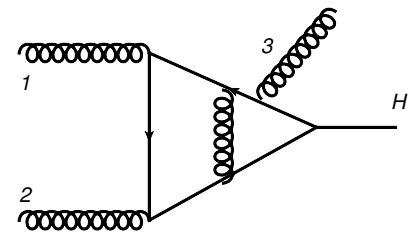

c)

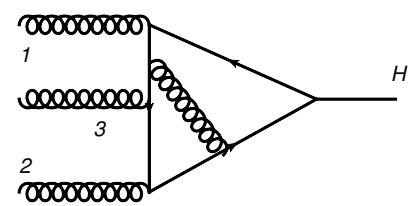

f)

Figure 2. Two-loop diagrams contributing to the abelian double logarithmic corrections. Diagrams that differ by the direction of the fermion flow are not shown.

vector contribution of the diagram figure $1 \mathrm{c}$ is given by the double logarithmic integral over the interval $|u| / s<\alpha<1, m_{b}^{2} /|t|<\beta<1$ from region I. It reads

$$
A_{++ \pm}^{(0), 1 c, v}= \pm L^{2} \int_{0}^{1-\tau_{u}} \mathrm{~d} \eta \int_{0}^{\tau_{t}-\eta} \mathrm{d} \xi=\mp L^{2} \frac{\left(1-\tau_{u}\right)\left(1-2 \tau_{t}-\tau_{u}\right)}{2} .
$$

We are now in position to present the leading-order bottom-quark contribution to $g g \rightarrow H g$ helicity amplitudes in the double logarithmic approximation. We sum the contributions of individual diagrams given in eqs. (3.8), (3.12), (3.21), (3.22) and obtain

$$
A_{+++}^{(0)}=L^{2}\left(1-\frac{\tau^{2}}{2}\right), \quad A_{++-}^{(0)}=-L^{2}\left(1+\frac{\tau^{2}}{2}\right)
$$

where we used $\tau=\ln \left(m_{b}^{2} / p_{\perp}^{2}\right) / L$. These results coincide with the double logarithmic limits of the one-loop amplitudes computed in ref. [19] long time ago. ${ }^{5}$ Our analysis identifies the origin of the double logarithmic enhancement of the $g g \rightarrow H g$ amplitude mediated by a light quark. With this understanding, it is straightforward to extend the above calculation first to two loops and then to all orders in the strong coupling constant $\alpha_{s}$. We will describe how to do this in the next sections.

\section{Two-loop helicity amplitudes in the double logarithmic approximation}

It is easy to convince oneself that a two-loop diagram contributing to $g g \rightarrow H g$ can develop leading $\mathcal{O}\left(m_{b}\right)$ double logarithmic enhancement only if exactly one of its fermion lines is soft. Indeed, since each soft fermion effectively contributes one power of $m_{b}$ to the final result, leading $\mathcal{O}\left(m_{b}\right)$ double logarithms are provided by exchanges of one soft fermion and one soft virtual gluon.

\footnotetext{
${ }^{5}$ See also ref. [18] for a recent discussion.
} 
The abelian part of the two-loop correction originates from a soft gluon exchange between virtual bottom quarks. The relevant two-loop Feynman diagrams are shown in figure 2. We note that the one-loop correction to $q \bar{q} H$ vertex appears as a subdiagram in many two-loop diagrams in figure 2. This correction develops a double logarithmic enhancement, so that the (properly normalized) $q \bar{q} H$ vertex in the one-loop approximation reads [15]

$$
V_{q \bar{q} H}=1+\delta V_{q \bar{q} H}, \quad \delta V_{q \bar{q} H}=-\frac{C_{F} \alpha_{s}}{2 \pi} \ln \left(\frac{q_{1}^{2}}{2 q_{1} \cdot q_{2}}\right) \ln \left(\frac{q_{2}^{2}}{2 q_{1} \cdot q_{2}}\right) .
$$

In eq. (4.1), $q_{1}$ and $q_{2}$ are the momenta of the off-shell quark lines and we assume that $m_{b}^{2} \ll q_{1}^{2}, q_{2}^{2} \ll q_{1} \cdot q_{2}$. This expression and the one-loop analysis of the previous section can be used to easily compute the leading logarithmic part of the relevant two-loop diagrams.

We begin with the diagram figure 2a. The external momenta of the vertex subgraph in this case are $q_{1}=p_{1}-l$ and $q_{2}=p_{2}+l$, where $l$ is the soft momentum of the quark loop. For $l=\alpha p_{1}+\beta p_{2}+l_{\perp}$ we get $q_{1}^{2}=s \beta, q_{2}^{2}=s \alpha, 2 q_{1} \cdot q_{2} \approx s$, so that

$$
\delta V_{q \bar{q} H}^{2 a}=-\frac{C_{F} \alpha_{s}}{2 \pi} \ln \alpha \ln \beta=-x \eta \xi,
$$

where $x=C_{F} \alpha_{s} L^{2} / 2 \pi$ and $\eta, \xi$ and $L$ are defined in the previous section. The double logarithmic integration over the soft quark momentum is the same as for the diagram figure 1a and the correction to the helicity amplitudes is obtained by including $\delta V_{q \bar{q} H}^{2 a}$ factor into the integrand of the one-loop expression eq. (3.8). For the two-loop abelian coefficient in eq. (2.6) we obtain

$$
A_{++ \pm}^{(1 A), 2 a}=\mp 2 L^{4} \int_{0}^{1-\tau_{t}} \mathrm{~d} \eta \int_{0}^{1-\eta} \mathrm{d} \xi \eta \xi=\mp L^{4} \frac{\left(1-4 \tau_{t}^{3}+3 \tau_{t}^{4}\right)}{12} .
$$

The diagram in figure $2 \mathrm{~b}$ is computed in a similar way. Virtualities of the quark lines become $q_{1}^{2} \approx|t|$ and $q_{2}^{2} \sim s \alpha$, and the one-loop vertex reads

$$
\delta V_{q \bar{q} H}^{2 b}=-\frac{C_{F} \alpha_{s}}{2 \pi} \ln \frac{|t|}{s} \ln \alpha=-x\left(1-\tau_{t}\right) \xi .
$$

Substituting this result into eq. (3.12), we obtain

$$
A_{++ \pm}^{(1 A), 2 b}=\mp L^{4} \int_{1-\tau_{t}}^{1} \mathrm{~d} \eta \int_{0}^{1-\eta} \mathrm{d} \xi\left(1-\tau_{t}\right) \xi=\mp L^{4} \frac{\left(\tau_{t}^{3}-\tau_{t}^{4}\right)}{6} .
$$

The double logarithmic contribution of the diagram figure $2 \mathrm{c}$ is generated when the quark propagator between the emission vertex of the soft gluon $g_{3}$ and the $q \bar{q} H$ vertex becomes independent of the soft photon loop momenta. In this case the inner loop reduces to the one-loop vertex with an additional restriction on the integration region. We find

$$
\delta V_{q \bar{q} H}^{2 c}=-\frac{C_{F} \alpha_{s}}{2 \pi} \ln \frac{\beta s}{|t|} \ln \alpha=-x\left(\eta-1+\tau_{t}\right) \xi .
$$

The corresponding two-loop corrections to the amplitudes read

$$
A_{++ \pm}^{(1 A), 2 c}=\mp L^{4} \int_{1-\tau_{t}}^{1} \mathrm{~d} \eta \int_{0}^{1-\eta} \mathrm{d} \xi\left(\eta-1+\tau_{t}\right) \xi=\mp L^{4} \frac{\tau_{t}^{4}}{24} .
$$


To compute the diagram figure $2 \mathrm{~d}$ in the double logarithmic approximation, we again insert the expression for the $q \bar{q} H$ vertex eq. (4.1) into the one-loop diagram in figure 1c. As was explained in the previous section this diagram receives the double logarithmic contributions from two independent momentum regions. In region I we parametrize the soft momentum as $l=\alpha p_{1}+\beta p_{3}+l_{\perp}$ and find

$$
\delta V^{2 d}=-\frac{C_{F} \alpha_{s}}{2 \pi} \ln \frac{|t| \beta}{s} \ln \frac{|u|+\alpha s}{s} \rightarrow-\frac{C_{F} \alpha_{s}}{2 \pi}\left\{\begin{array}{cc}
\ln \frac{|t| \beta}{s} \ln \frac{|u|}{s} & \alpha<\frac{|u|}{s} \\
\ln \frac{|t| \beta}{s} \ln \alpha & \alpha>\frac{|u|}{s} .
\end{array}\right.
$$

We note that the two integration regions, $\alpha<|u| / s$ and $\alpha>|u| / s$, correspond to scalar and vector contributions, respectively. The expression for the $q \bar{q} H$ vertex in region II can be found in the same way. We insert these results into eqs. (3.21), (3.22) and obtain

$$
\begin{aligned}
A_{++-}^{(1 A), 2 d, s} & =L^{4} \int_{1-\tau_{u}}^{\tau_{t}} \mathrm{~d} \eta \int_{0}^{\tau_{t}-\eta} \mathrm{d} \xi\left(1-\tau_{u}\right)\left(\xi+1-\tau_{t}\right)+(t \leftrightarrow u) \\
& =L^{4} \frac{\left(2-2 \tau_{t}+\tau_{u}\right)\left(1-\tau_{u}\right)\left(1-\tau_{t}-\tau_{u}\right)^{2}}{6}+(t \leftrightarrow u) . \\
A_{++ \pm}^{(1 A), 2 d, v} & =\mp L^{4} \int_{0}^{1-\tau_{u}} \mathrm{~d} \eta \int_{0}^{\tau_{t}-\eta} \mathrm{d} \xi\left(\xi+1-\tau_{t}\right) \eta \\
& = \pm L^{4} \frac{\left(1-\tau_{u}\right)^{2}\left(5-12 \tau_{t}+6 \tau_{t}^{2}-2 \tau_{u}-3 \tau_{u}^{2}\right)}{24} .
\end{aligned}
$$

We note that the corresponding one-loop expression given in eq. (3.21) includes equal scalar contributions from regions I and II. At two loops, contributions of regions I and II are not equal anymore; we separate them in eq. (4.9) and indicate contribution of the region I by the corresponding integral and the contribution of region II by the $t \leftrightarrow u$ symmetric term. The vector contribution eq. (4.10) comes entirely from region I, as in the one-loop case.

Diagrams shown in figure $2 \mathrm{e}$ and figure $2 \mathrm{f}$ are related by crossing symmetry and we only consider the evaluation of the former. This diagram receives the scalar contribution from region I; the double logarithmic term is generated when the propagator between the emission vertex of the gluon $g_{2}$ and the $q \bar{q} H$ annihilation vertex becomes independent on the soft momenta. Thus as in the case of the diagram figure $2 \mathrm{c}$ the inner loop reduces to the one-loop vertex integral with an additional restriction on the integration region. The effective vertex in this case reads

$$
\delta V_{q \bar{q} H}^{2 e}=-\frac{C_{F} \alpha_{s}}{2 \pi} \ln \frac{\alpha s}{u} \ln \beta=-x\left(\eta-1+\tau_{u}\right) \xi .
$$

Since the scalar contribution to the all-plus helicity amplitude vanishes, we obtain

$$
A_{++-}^{(1 A), 2 e}=L^{4} \int_{1-\tau_{u}}^{\tau_{t}} \mathrm{~d} \eta \int_{0}^{\tau_{t}-\eta} \mathrm{d} \xi\left(\eta-1+\tau_{u}\right) \xi=L^{4} \frac{\left(1-\tau_{t}-\tau_{u}\right)^{4}}{24} .
$$

The result for the diagram in figure $2 \mathrm{f}$ is also given by eq. ( 4.12 ) since it is symmetric with respect to the replacement $t \leftrightarrow u$. 
Taking the sum of all the individual contributions in eqs. (4.3), (4.5), (4.7), (4.9), (4.10), (4.12), we obtain the two-loop correction to the $g g \rightarrow H g$ amplitude in the double logarithmic approximation

$$
\begin{aligned}
& A_{+++}^{(1 A)}=-\frac{L^{2}}{24}\left(2-3 \tau^{2}+2 \tau^{3}+3 \tau^{2} \zeta^{2}\right), \\
& A_{++-}^{(1 A)}=\frac{L^{2}}{24}\left(2+3 \tau^{2}-6 \tau^{3}+4 \tau^{4}-3 \tau^{2} \zeta^{2}\right),
\end{aligned}
$$

where the new variable $\zeta=\ln (u / t) / L$ parametrizes the dependence of the amplitudes on the soft gluon rapidity.

\section{Resummation of the abelian double logarithmic contributions}

The perturbative expansion parameter for the double logarithmic corrections $x=\frac{C_{F} \alpha_{s}}{2 \pi} L^{2}$ is not small numerically, $x \sim 1$. For this reason, resummation of such corrections might be relevant. This problem is also quite interesting theoretically, since very little is known about the all-order structure of the power-suppressed non-Sudakov logarithms. Indeed, on the one hand, only few examples of the resummation of non-Sudakov double logarithmic corrections are known so far [20-22] and, on the other hand, systematic renormalization group analysis of the high-energy behavior of the on-shell amplitudes beyond the leadingpower approximation is still elusive for existing effective field theory methods.

The problem that we discuss in this paper is, however, simpler than the general case. As we pointed out already, to leading order in $m_{b}$, higher-order double logarithmic corrections to the helicity amplitudes are caused by multiple soft virtual gluon exchanges and a single soft quark exchange. Thus we have to consider Feynman diagrams similar to figure 2 but with multiple soft gluon exchanges between different quark lines. For the abelian part of the corrections we can use simple factorization properties of soft emissions in QED. It is well-known that in this case, upon summing over all relevant diagrams, integrations over soft gluon momenta factorize and the all-order result is given by the exponent of the single gluon contribution, given by the $\mathcal{O}\left(\alpha_{s}\right)$ term in eq. (4.1).

By using the expression eq. (4.2) specific for the diagram figure 2a we find the Sudakov exponent to be $e^{-x \xi \eta}$. The all-order double logarithmic corrections to helicity amplitudes are then obtained by including this exponent into the integrand of eq. (3.8). Upon integration over $\xi$, we obtain the resummed expression for helicity amplitudes in the form of the one-parameter integral

$$
A_{++ \pm}^{\mathrm{A}, \mathrm{a}}= \pm 2 L^{2} \int_{0}^{1-\tau_{t}} \frac{1-e^{-x \eta(1-\eta)}}{x \eta} \mathrm{d} \eta .
$$

The multiple gluon exchange diagrams related to figure $2 \mathrm{~b}$ and figure $2 \mathrm{c}$ must be considered simultaneously. ${ }^{6}$ After summing over all possible permutations of the soft gluon emission vertices, their contributions factorize and produce a product of the exponents of the oneloop contributions (4.4), (4.6). They combine into the exponential factor $e^{-x \xi \eta}$ identical to

\footnotetext{
${ }^{6}$ Indeed, already at the two-loop level, these diagrams describe the two possible ways to emit the soft gluon with momentum $p_{3}$ and a soft virtual gluon by an energetic quark line.
} 
the previous case. By including it into the integrand of the one-loop expression eq. (3.12) we get the all-order result

$$
A_{++ \pm}^{\mathrm{A}, \mathrm{bc}}= \pm L^{2} \int_{1-\tau_{t}}^{1} \frac{1-e^{-x \eta(1-\eta)}}{x \eta} \mathrm{d} \eta .
$$

For the diagram figure $2 \mathrm{~d}$ the Sudakov factor depends on whether a vector or a scalar contribution is considered, cf. eq. (4.8). For the vector part, the Sudakov exponent is $e^{-x\left(\xi+1-\tau_{t}\right) \eta}$ and the all-order result associated with the leading-order contribution eq. (3.22) reads

$$
A_{++ \pm}^{\mathrm{A}, \mathrm{d}, \mathrm{v}}= \pm L^{2} \int_{0}^{1-\tau_{u}} \frac{e^{-x\left(1-\tau_{t}\right) \eta}-e^{-x \eta(1-\eta)}}{x \eta} \mathrm{d} \eta .
$$

As in the case of the diagrams figure $2 \mathrm{~b}$ and figure $2 \mathrm{c}$, the scalar contributions from the momentum region I of the diagram figure $2 \mathrm{~d}$ combine with the diagram figure $2 \mathrm{e}$, exponentiate and produce a Sudakov factor $e^{-x\left(1-\tau_{t}-\tau_{u}+\tau_{t} \tau_{u}+\eta \xi\right)}$. The Sudakov exponent of the scalar contribution from region II of the diagrams that combine figure $2 \mathrm{~d}$ and figure $2 \mathrm{e}$ with additional soft exchanges is obtained by the replacement $t \leftrightarrow u$. The sum of these contributions is, therefore, given by

$$
A_{++-}^{\mathrm{A}, \mathrm{de}, \mathrm{s}}=-2 L^{2} \int_{1-\tau_{u}}^{\tau_{t}} \frac{e^{-x\left(1-\tau_{u}\right)\left(1-\tau_{t}\right)}\left(1-e^{-x \eta\left(\tau_{t}-\eta\right)}\right)}{x \eta} \mathrm{d} \eta+(t \leftrightarrow u) .
$$

The sum of individual contributions given in eqs. (5.1), (5.2), (5.3), (5.4) determines the complete result for the abelian double logarithmic corrections to the bottom quark contribution to $g g \rightarrow H g$ helicity amplitudes to all orders in QCD perturbation theory.

\section{Double logarithmic corrections to the differential cross section}

We are now in position to estimate the effect of the corrections, computed in the previous section, on the differential cross section of the Higgs boson production in association with a jet. The total amplitude of this process is given by the sum of top and bottom contributions since contributions of lighter quarks are negligible. We therefore write

$$
\begin{aligned}
& M_{+++}^{\text {soft }}=-g_{s} \sqrt{2} f^{a_{1} a_{2} a_{3}} \frac{g_{s}^{2}}{16 \pi^{2} v} \frac{\langle 12\rangle^{2}}{[12]\langle 23\rangle\langle 13\rangle}\left[A_{+++}^{(t)}+\frac{m_{b}^{2}}{m_{H}^{2}} A_{+++}^{(b)}\right], \\
& M_{++-}^{\text {soft }}=-g_{s} \sqrt{2} f^{a_{1} a_{2} a_{3}} \frac{g_{s}^{2}}{16 \pi^{2} v} \frac{\langle 12\rangle}{[23][13]}\left[A_{++-}^{(t)}+\frac{m_{b}^{2}}{m_{H}^{2}} A_{++-}^{(b)}\right] .
\end{aligned}
$$

Thanks to its large Yukawa coupling, the top quark provides the dominant contribution to the scattering amplitude. In the soft limit the real emission from inside the top-quark loop is power-suppressed i.e. the soft emission factorizes with respect to the $g g \rightarrow H$ amplitude. The result for this contribution is well known and in the limit of an infinitely heavy top quark reads

$$
A_{++ \pm}^{(t)}= \pm \frac{2}{3}
$$

There are $\mathcal{O}\left(\alpha_{s}\right)$ corrections to this formula that, however, are not essential for us. 
The largest effect of the bottom quark on the differential cross section is caused by its interference with the top quark contribution. We find

$$
d \sigma_{g g \rightarrow H g}=d \sigma_{g g \rightarrow H g}^{(0)} \times\left[1-\frac{3}{2} \frac{m_{b}^{2}}{m_{H}^{2}}\left(A_{+++}^{(b)}-A_{++-}^{(b)}\right)+\mathcal{O}\left(m_{b}^{4}\right)\right],
$$

where $d \sigma_{g g \rightarrow H g}^{(0)}$ is the top quark mediated cross section, and we neglect the finite top mass effects in the interference term. Note that since the leading bottom quark effect is due to the interference with the top quark mediated amplitude, to leading order in $1 / m_{t}$, any additional real emission contribution involves the three-gluon interaction and does not contribute to the abelian part of the correction.

We can now use the result derived in the previous section for numerical estimates. It is convenient to express the correction to the cross section through the variables $\tau=$ $\ln \left(m_{b}^{2} / p_{\perp}^{2}\right) / L$ and $\zeta=\ln (u / t) / L$, which parameterize the dependence of the cross section on the transverse momentum and rapidity. We obtain

$$
d \sigma_{g g \rightarrow H g}=d \sigma_{g g \rightarrow H g}^{(0)} \times\left[1-\frac{3}{2} \frac{m_{b}^{2}}{M_{H}^{2}} L^{2} f(x, \tau, \zeta)+\mathcal{O}\left(m_{b}^{4}\right)\right],
$$

where

$$
\begin{aligned}
x f(x, \tau, \zeta)= & \int_{0}^{1} \frac{\mathrm{d} \eta}{\eta}\left[\left(1-e^{-x \eta(1-\eta)}\right)(1+2 \theta(1-\tau-\zeta-2 \eta))-\left(1-e^{-x \eta \delta(\tau, \zeta)}\right)\right] \\
& +e^{-x \delta(\tau, \zeta)} \int_{(1-\tau+\zeta) / 2}^{(1+\tau+\zeta) / 2} \frac{\mathrm{d} \eta}{\eta}\left(1-e^{-x \eta(1+\tau+\zeta-2 \eta) / 2}\right)+(\zeta \rightarrow-\zeta),
\end{aligned}
$$

and $\delta(\tau, \zeta)=\left((1-\tau)^{2}-\zeta^{2}\right) / 4$. The perturbative expansion of the function $f$ reads

$$
f=2-\frac{x}{6}\left(1-\tau^{3}+\tau^{4}\right)+\frac{x^{2}}{24}\left(\frac{4}{15}-\tau^{3}+2 \tau^{4}-\frac{7 \tau^{5}}{5}+\frac{2 \tau^{6}}{5}+\zeta^{2}\left(\tau^{3}-\tau^{4}\right)\right)+\ldots
$$

where ellipsis stands for terms suppressed by higher powers of $x$.

We can use the result eq. (6.6) to estimate the impact of the QCD corrections to bottom quark contributions to $g g \rightarrow H g$ on the Higgs boson transverse momentum distribution. In principle, we should convolute the partonic cross section eq. (6.4) with the parton distribution functions. However, we will now argue that, given the structure of the corrections shown in eq. (6.6), this is not necessary. Indeed, within the accuracy of our approximation $L=\ln \left(s / m_{b}^{2}\right) \approx \ln \left(m_{H}^{2} / m_{b}^{2}\right)$ can be considered independent of the partonic center-of-mass energy. In addition, the series in eq. (6.6) shows very weak dependence on the rapidity of the soft gluon. Indeed, the function $f$ in eq. (6.6) does not depend on the gluon rapidity up to $\mathcal{O}(x)$. Moreover, at $\mathcal{O}\left(x^{2}\right)$ the rapidity-dependent part of the coefficient includes only high powers of $\tau$. If the soft gluon is emitted at large rapidity, $|\zeta| \approx 1$ and $\tau \ll 1$. On the contrary, central emission with the large transverse momentum implies $|\zeta| \ll 1$ and $\tau \approx 1$. Therefore, the rapidity-dependent term is small everywhere and can be neglected. After these modifications the function $f$ depends only on the transverse momentum of the emitted gluon or the Higgs boson. As a result it remains unaffected by 
the integration over parton distribution functions if the transverse momentum of the Higgs boson is kept fixed. Therefore, we can write

$$
\begin{aligned}
\frac{d \sigma_{p p \rightarrow H+j}}{d p_{\perp}^{2}}=\frac{d \sigma_{p p \rightarrow H+j}^{(0)}}{d p_{\perp}^{2}}\left\{1-\frac{3 m_{b}^{2}}{m_{H}^{2}} L_{\mathrm{eff}}^{2}\left[1-\frac{x_{\mathrm{eff}}}{12}\left(1-\tau^{3}+\tau^{4}\right)\right.\right. \\
\left.\left.+\frac{x_{\mathrm{eff}}^{2}}{48}\left(\frac{4}{15}-\tau^{3}+2 \tau^{4}-\frac{7 \tau^{5}}{5}+\frac{2 \tau^{6}}{5}\right)+\mathcal{O}\left(x_{\mathrm{eff}}^{3}\right)\right]+\mathcal{O}\left(m_{b}^{4}\right)\right\}
\end{aligned}
$$

where $L_{\text {eff }}=\ln \left(m_{H}^{2} / m_{b}^{2}\right)$ and $x_{\text {eff }}=\frac{\alpha_{S} C_{F}}{2 \pi} L_{\text {eff }}^{2}$. We emphasize that eq. (6.7) only applies to the contribution of $g g$ partonic channel to the production of the Higgs boson in proton collisions and that only abelian corrections are taken into account there.

We note that the series in eq. (6.7) has peculiar structure. Indeed, the one-loop double logarithmic correction to $\mathrm{d} \sigma / \mathrm{d} p_{\perp}$ is independent on $p_{\perp}$, thanks to a cancellation between $p_{\perp}$-dependent contributions to individual helicity amplitudes eq. (3.23), when the differential cross section is evaluated [18]. In principle, it could have been possible to interpret this result as an indication that the naive factorization of soft emissions extends to a region beyond $p_{\perp}>m_{b}$, at least inasmuch as the interference with the top quark loop is concerned. However, our result eq. (6.7) shows that such an interpretation does not hold and that the cancellation of $p_{\perp}$-dependent double logarithmic corrections does not persist beyond one-loop. In fact, starting from three loops, the double logarithmic corrections to the differential cross section start to depend on the rapidity of the emitted gluon as eq. (6.6) shows.

To understand the numerical impact of these corrections, we use $m_{H}=125 \mathrm{GeV}$, $m_{b}=4.2 \mathrm{GeV}, \alpha_{s}=0.12$ and consider $p_{\perp}$ in the range $m_{b}<p_{\perp}<50 \mathrm{GeV}$. We note that the one-loop double logarithmic corrections reduce the cross section by about $16 \%$. This is somewhat larger than the result of the full computation, but still in the right ballpark. The two-loop correction increases the result by about $1.5 \%$. This is somewhat smaller than the next-to-leading order effect in $g g \rightarrow H$ cross section but, given the fact that we only consider the abelian contribution here, the two results are not inconsistent. ${ }^{7}$ However, our main interest is in $p_{\perp}$-dependent corrections and these corrections turn out to be quite small, see figure 3. In fact, the two-loop correction in eq. (6.7) decreases by just about $0.2 \%$ when the transverse momentum varies from $p_{\perp} \sim m_{b}$ to $p_{\perp} \sim 50 \mathrm{GeV}$. This tiny change is the result of a strong cancellation between $\tau^{3}$ and $\tau^{4}$ term in eq. (6.7). When taken separately, these terms could have caused a change in the two-loop result that is closer to one percent. The three-loop correction in eq. (6.7) changes the prediction by about $-0.1 \%$ and its $p_{\perp}$-dependent part is one order of magnitude smaller.

\section{Conclusion}

In this paper, we have studied the bottom-quark loop contribution to the production of the Higgs boson in association with a jet in gluon fusion in the double logarithmic

\footnotetext{
${ }^{7}$ The top-bottom interference changes the $m_{t} \rightarrow \infty$ inclusive cross section by approximately $-12 \%$ at leading order. QCD corrections to the bottom loop decrease this leading order result by fifty percent.
} 


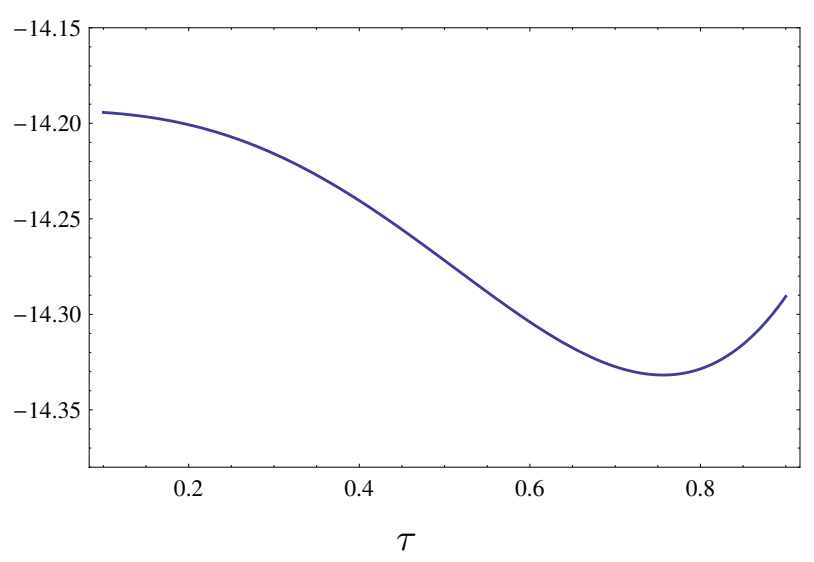

Figure 3. The bottom-quark loop corrections to the Higgs boson transverse momentum distribution, eq. (6.7), in percent to the leading top-quark loop contribution as function of the variable $\tau=\ln \left(m_{b}^{2} / p_{\perp}^{2}\right) / \ln \left(m_{b}^{2} / m_{h}^{2}\right)$. The values of the input parameters are specified in the text. The transverse momentum dependence of the corrections is numerically dominated by the two-loop term.

approximation. This contribution is suppressed by the ratio of the bottom-quark mass to the Higgs boson mass but, at the same time, it is enhanced by two powers of large logarithms, $\ln \left(s / m_{b}^{2}\right)$ or $\ln \left(p_{\perp}^{2} / m_{b}^{2}\right)$, per one power of the strong coupling constant. As it is repeatedly emphasized in the literature, these terms may be important for phenomenology, in particular for the description of the Higgs boson transverse momentum distribution in an interesting kinematic region $m_{b}<p_{\perp}<m_{H}$. We have analyzed the abelian part of the double logarithmic corrections and computed the $g g \rightarrow H g$ helicity amplitudes which incorporate these terms to all orders in $\alpha_{s}$.

Numerically, the abelian corrections appear to be moderate. For example, the two-loop corrections change the transverse momentum distribution by about two percent. However it is important to note that the $p_{\perp}$-dependent part of these corrections is only about $0.2 \%$ due to the cancellation between different $p_{\perp}$-dependent terms. Assuming that, up to an obvious change in the color factor $C_{F} \rightarrow C_{A}$, the non-abelian corrections will be similar to the abelian ones, we estimate the yet unknown non-abelian corrections to be about three times larger. We conclude that the description of the Higgs boson transverse momentum distribution with a few percent precision requires a calculation of the $\mathcal{O}\left(\alpha_{s}\right)$ logarithmically enhanced non-abelian corrections to bottom quark contribution while the all-order resummation is, probably, not important. Our analysis sets up a framework for such a calculation. A new element in the calculation of the non-abelian part is its infra-red sensitivity and a related need to account for the contribution of soft radiation.

\section{Acknowledgments}

K.M. would like to thank F. Caola for useful conversations. The work of A.P. is supported in part by NSERC and Perimeter Institute of Theoretical Physics. 


\section{A Polarization vectors}

The initial state gluon with momentum $k$ and the gauge vector $r$ is described by the following polarization vectors

$$
\epsilon_{+}^{\mu}=-\frac{\left[r \gamma^{\mu} k\right\rangle}{\sqrt{2}[r k]}, \quad \epsilon_{-}^{\mu}=\frac{\left\langle r \gamma^{\mu} k\right]}{\sqrt{2}\langle r k\rangle} .
$$

The polarization vectors for the final state gluon are obtained by exchanging $\epsilon_{+} \leftrightarrow \epsilon_{-}$.

As reference vectors, we choose $p_{1,2}$ for $\epsilon_{2,1}$ and $p_{2}$ for $\epsilon_{3}$. The latter choice allows us to ignore all the contributions where the soft gluon $g_{3}$ is emitted by either gluon $g_{2}$ or a fermion that carries momentum $p_{2}$. The full list of polarization vectors that we use in the calculation, with all the reference vectors explicitly shown, reads

$$
\begin{array}{rlrl}
\epsilon_{+}^{\mu}(1) & =-\frac{1}{\sqrt{2}} \frac{\left[2 \gamma^{\mu} 1\right\rangle}{[21]}, & \epsilon_{-}^{\mu}(1) & =\frac{1}{\sqrt{2}} \frac{\left\langle 2 \gamma^{\mu} 1\right]}{\langle 21\rangle}, \\
\epsilon_{+}^{\mu}(2)=-\frac{1}{\sqrt{2}} \frac{\left[1 \gamma^{\mu} 2\right\rangle}{[12]}, & \epsilon_{-}^{\mu}(2)=\frac{1}{\sqrt{2}} \frac{\left\langle 1 \gamma^{\mu} 2\right]}{\langle 12\rangle}, \\
\epsilon_{+}^{\mu}(3)=\frac{1}{\sqrt{2}} \frac{\left\langle 2 \gamma^{\mu} 3\right]}{\langle 23\rangle}, & \epsilon_{-}^{\mu}(3)=-\frac{1}{\sqrt{2}} \frac{\left[2 \gamma^{\mu} 3\right\rangle}{[23]} .
\end{array}
$$

Open Access. This article is distributed under the terms of the Creative Commons Attribution License (CC-BY 4.0), which permits any use, distribution and reproduction in any medium, provided the original author(s) and source are credited.

\section{References}

[1] ATLAS collaboration, Observation of a new particle in the search for the Standard Model Higgs boson with the ATLAS detector at the LHC, Phys. Lett. B 716 (2012) 1 [arXiv: 1207.7214] [INSPIRE].

[2] CMS collaboration, Observation of a new boson at a mass of $125 \mathrm{GeV}$ with the CMS experiment at the LHC, Phys. Lett. B 716 (2012) 30 [arXiv:1207.7235] [INSPIRE].

[3] C. Anastasiou, C. Duhr, F. Dulat, F. Herzog and B. Mistlberger, Higgs boson gluon-fusion production in QCD at three loops, Phys. Rev. Lett. 114 (2015) 212001 [arXiv:1503.06056] [INSPIRE].

[4] C. Arnesen, I.Z. Rothstein and J. Zupan, Smoking guns for on-shell new physics at the LHC, Phys. Rev. Lett. 103 (2009) 151801 [arXiv:0809.1429] [INSPIRE].

[5] E. Bagnaschi, G. Degrassi, P. Slavich and A. Vicini, Higgs production via gluon fusion in the POWHEG approach in the SM and in the MSSM, JHEP 02 (2012) 088 [arXiv:1111.2854] [INSPIRE].

[6] A. Azatov and A. Paul, Probing Higgs couplings with high $p_{T}$ Higgs production, JHEP 01 (2014) 014 [arXiv:1309.5273] [inSPIRE].

[7] E. Bagnaschi and A. Vicini, The Higgs transverse momentum distribution in gluon fusion as a multiscale problem, JHEP 01 (2016) 056 [arXiv: 1505.00735] [INSPIRE].

[8] U. Langenegger, M. Spira and I. Strebel, Testing the Higgs boson coupling to gluons, arXiv: 1507.01373 [INSPIRE]. 
[9] R. Boughezal, F. Caola, K. Melnikov, F. Petriello and M. Schulze, Higgs boson production in association with a jet at next-to-next-to-leading order, Phys. Rev. Lett. 115 (2015) 082003 [arXiv: 1504.07922] [INSPIRE].

[10] R. Boughezal, C. Focke, W. Giele, X. Liu and F. Petriello, Higgs boson production in association with a jet at NNLO using jettiness subtraction, Phys. Lett. B 748 (2015) 5 [arXiv: 1505.03893] [INSPIRE].

[11] D. de Florian, G. Ferrera, M. Grazzini and D. Tommasini, Transverse-momentum resummation: Higgs boson production at the Tevatron and the LHC, JHEP 11 (2011) 064 [arXiv: 1109.2109] [INSPIRE].

[12] T. Becher, M. Neubert and D. Wilhelm, Higgs-boson production at small transverse momentum, JHEP 05 (2013) 110 [arXiv:1212.2621] [INSPIRE].

[13] D. Neill, I.Z. Rothstein and V. Vaidya, The Higgs transverse momentum distribution at NNLL and its theoretical errors, JHEP 12 (2015) 097 [arXiv: 1503.00005] [INSPIRE].

[14] R. Mueller and D.G. Oeztuerk, On the computation of finite bottom-quark mass effects in Higgs boson production, arXiv:1512.08570 [INSPIRE].

[15] V.V. Sudakov, Vertex parts at very high-energies in quantum electrodynamics, Sov. Phys. JETP 3 (1956) 65 [Zh. Eksp. Teor. Fiz. 30 (1956) 87] [INSPIRE].

[16] H. Mantler and M. Wiesemann, Top- and bottom-mass effects in hadronic Higgs production at small transverse momenta through LO+NLL, Eur. Phys. J. C 73 (2013) 2467 [arXiv: 1210.8263 ] [INSPIRE].

[17] M. Grazzini and H. Sargsyan, Heavy-quark mass effects in Higgs boson production at the LHC, JHEP 09 (2013) 129 [arXiv:1306.4581] [INSPIRE].

[18] A. Banfi, P.F. Monni and G. Zanderighi, Quark masses in Higgs production with a jet veto, JHEP 01 (2014) 097 [arXiv: 1308.4634] [INSPIRE].

[19] U. Baur and E.W.N. Glover, Higgs boson production at large transverse momentum in hadronic collisions, Nucl. Phys. B 339 (1990) 38 [INSPIRE].

[20] V.G. Gorshkov, V.N. Gribov, L.N. Lipatov and G.V. Frolov, Doubly logarithmic asymptotic behavior in quantum electrodynamics, Sov. J. Nucl. Phys. 6 (1968) 95 [Yad. Fiz. 6 (1967) 129] [INSPIRE].

[21] M.I. Kotsky and O.I. Yakovlev, On the resummation of double logarithms in the process Higgs $\rightarrow \gamma \gamma$, Phys. Lett. B 418 (1998) 335 [hep-ph/9708485] [INSPIRE].

[22] A.A. Penin, High-energy limit of quantum electrodynamics beyond Sudakov approximation, Phys. Lett. B $\mathbf{7 4 5}$ (2015) 69 [Erratum ibid. B $\mathbf{7 5 1}$ (2015) 596] [arXiv:1412.0671] [INSPIRE]. 